\title{
El masaje reflexológico podal como medio alternativo en el tratamiento de adultos hipertensos
}

\author{
Foot reflexology massage as an alternative means of treating hypertensive \\ adults
}

MSc. Eugenio Rodolfo González Pérez. ${ }^{1}$ \& Lic Arcelia González Paneque. ${ }^{2}$

Recibido: 05-01-2018 / Revisado: 06-02-2018 / Aceptado: 07-03-2018 / Publicado: 05-04-2018

DOI: https://doi.org/10.33262/anatomiadigital.v1i1.1037

\begin{abstract}
.
The advances experienced in the science and the technique, they have transformed to the XXI century into the century of the information that which has their repercussion in the field of the Therapeutic Physical Culture. Therapies of manipulation of the therapeutic massage have been introduced that allow to generate, to solve and to exchange information about the result in their use of these therapies, being promoted a significant socialization of the use of the same ones, to which is not other people's the education. The work arises starting from experiences in the application of the massage Reflexológico Podal in the treatment of mature active hipertensos linked to programs of the Community Physical Activity of the
\end{abstract}

\section{Resumen.}

Los avances experimentados en la ciencia y la técnica han convertido al siglo XXI en el siglo de la información lo cual tiene su repercusión en el campo de la Cultura Física Terapéutica. Se han introducido terapias de manipulación del masaje terapéutico que permiten generar, resolver e intercambiar información acerca del resultado en su uso de estas terapias, promoviéndose una socialización significativa del uso de las mismas, a la cual no está ajena la educación. El trabajo surge a partir de experiencias en la aplicación del masaje Reflexológico Podal en el tratamiento de adultos hipertensos activos vinculados a programas de la Actividad Física Comunitaria del municipio Güines Provincia Mayabeque que desde la

${ }^{1}$ Ciencias Médicas Mayabeque, Departamento Cultura Física jefe de Departamento, Mayabeque, Cuba, ergonzales@infomed.sld.cu iD https://orcid.org/0000-0002-7353-0180

2 Ciencias Médicas Mayabeque, Departamento Ciencias Biológicas, Mayabeque, Cuba, aggonzales@infomed.sld.cu iD https://orcid.org/0000-0002-7353-0181 
municipality Güines County Mayabeque that from the prophylaxis and therapy constitutes an important base to eliminate signs and symptoms of the illness elevating the functional physical state. The same one has as objective to expose the results of a treatment that it has been applied, sophisticated and used in investigations during several years for the authors and other professionals of the specialty, starting from the knowledge acquired in the work prophylaxis and therapy. In the development of the work the methodology is exposed for the application of the massage reflexológico podal, Steps of Relaxation. Starting from these results you could conclude that the massage influenced positively in the treatment of the HTA in the adult, symptoms found its disappearance with this treatment type achieving high motivation levels and satisfaction for the results in the investigated fellows.

Keywords: Podic reflexology, Hypertension. profiláctica y terapéutica constituye una importante base para eliminar signos $\mathrm{y}$ síntomas de la enfermedad elevando el estado físico funcional. El mismo tiene como objetivo aplicar la Reflexología podal a hipertensos adultos activos vinculados al programa Actividad Física comunitaria. En el desarrollo del trabajo se expone la metodología para la aplicación del masaje reflexológico podal, Pasos de Relajación. A partir de estos resultados se pudo concluir que el masaje influyó positivamente en el tratamiento de la HTA en el adulto, síntomas encontraron su desaparición con este tipo de tratamiento logrando altos niveles de motivación y satisfacción por los resultados en los sujetos investigados.

\section{Introducción.}

La Hipertensión arterial en el Siglo XXI, es la más común de las enfermedades que afecta la salud de los individuos de poblaciones en todas partes del mundo por lo que representa por sí misma una enfermedad constituyendo un factor de riesgo importante para otras enfermedades.

La enfermedad cardiovascular se está convirtiendo en la mayor causa de morbilidad y mortalidad, tanto en los países desarrollados como en los subdesarrollados. La hipertensión arterial es el factor de riesgo más importante para esta nueva epidemia y determinante principal del incremento de enfermedad cerebro vascular, infarto agudo del miocardio, Insuficiencia Renal Crónica, insuficiencia cardiaca congestiva y de enfermedad vascular periférica (Atark 2005). Aunque se ha avanzado en el conocimiento del tratamiento farmacológico y no farmacológico y a pesar del énfasis que se ha hecho en la aplicación de estas medidas al nivel poblacional, con frecuencia la hipertensión es tratada inadecuadamente lo cual contribuye de modo significativo al panorama actual que las enfermedades cardiovasculares presentan al nivel mundial y en nuestro país. 
En Cuba la prevalencia de HTA es de $30 \%$ en zonas urbanas y $15 \%$ en la rural, existe el criterio de que cifras de presiones diastólicas aumentan considerablemente con la edad (Gifford RW. 2005)

La hipertensión lesiona fundamentalmente 4 órganos.

1.-. Corazón. Aterosclerosis coronaria con angina de pecho, o infarto del miocardio. Hipertrofia ventricular izquierda.

Insuficiencia cardíaca.

Edema agudo del pulmón.

2.- Vasos. Aneurisma disecante de la aorta.

Arteriolosclerosis.

3.- Riñón. Arteriosclerosis, nefroanglosclerosis, insuficiencia renal. Hipertensión maligna o en fase acelerada.

4.- Cerebro. Hemorragia cerebral, trombosis cerebral, hemorragia subaracnoidea. Las alteraciones anatómicas que produce en estos se deben a las lesiones vasculares que se presentan en la hipertensión. MINSAP (1998)

\section{Desarrollo}

Se realizó un estudio de tipo cuasi-experimental en pacientes con hipertensión arterial activos vinculados a programas de la Actividad Física Comunitaria del municipio Güines, en el período de enero de 2017 a febrero de 2017, con el propósito de demostrar la influencia del masaje reflexológico podal en estos pacientes.

Los métodos empleados fueron: análisis y síntesis, histórico lógico, inducción deducción, revisión documental, encuesta, observaciones a clases, estadístico-matemático.

\section{Población y muestra}

31 pacientes hipertensos activos vinculados a programas de la Actividad Física Comunitaria del municipio Güines y la muestra de estudio estuvo representada por 9 pacientes, que constituyen un $29 \%$ de la población; ésta fue seleccionada mediante muestreo aleatorio simple. A los pacientes se les pidió su voluntariedad para incluirlos en la investigación, con lo cual se respetó el principio bioético de autonomía. Para la obtención de la información se utilizaron las historias clínicas de los pacientes, donde se identificó edad, sexo, raza y tipo de hipertensión. La tarjeta de control nos proporcionó determinar el comportamiento de la hipertensión arterial antes de aplicado el tratamiento y después del mismo. 
Para desarrollar la investigación se siguieron los siguientes pasos metodológicos:

- Búsqueda bibliográfica

- Análisis documental

- Estudio y determinación de la población y la muestra para el diagnóstico

- Elaboración de los instrumentos y adecuación en correspondencia con las particularidades de los adultos y el conocimiento de los profesores para de esta forma conocer la pertinencia de la aplicación.

- Elaboración de las técnicas de manipulación para la hipertensión arterial

- Aplicación de los instrumentos del diagnóstico

- Análisis e interpretación de los resultados obtenidos.

La presente investigación estuvo conformada, de manera general, por tres etapas: etapa de diagnóstico, selección y elaboración de las técnicas de manipulación y etapa de validación de las técnicas relacionando en todo momento los resultados cualitativos y cuantitativos de manera dialéctica. En opinión de los autores de esta investigación, estas etapas son la que mejor se relacionan con las condiciones y características de los adultos hipertensos que realmente estén interesados en lograr niveles superiores de calidad de vida.

a) Etapa de diagnóstico: Se realizaron encuestas a los adultos y técnicos de cultura física, con el fin de constatar las necesidades de mejorar y elevar el estado funcional en los adultos mayores hipertensos de la casa de los abuelos.

b) Para la elaboración de las técnicas de manipulación para la hipertensión arterial se siguió la siguiente secuencia.

Fundamentos teóricos de las técnicas del sistema del pie y la reflexología, su concepto, historia, vínculos de su estimulación con la salud individual, de la familia y la comunidad.

El sistema del pie y la reflexología. Aplicaciones de la reflexología en los distintos componentes del sistema del pie.

Repercusiones de la reflexología del pie en el sistema del cuerpo humano.

c) Validación de las técnicas

\section{Pasos a seguir para bajar o subir la presión sanguínea}

Cada sesión será de 15 o 20 minutos, tres veces por semana

1. Aplique a ambos pies las técnicas de relajación y termine con la presión del pulgar en el plexo solar de los dos .Relaje cada pie separadamente.

2. Pasee el pulgar por las cincos zonas de un pie y trabaje los puntos señalados 
3. Pasee el pulgar por las cincos zonas del otro pie y trabaje los puntos señalados

4. Integre las técnicas de relajación cuantas veces le sean necesarias a su receptor, para relajarlo cuando se encuentre usted con zonas delicadas

\section{Encuesta a Técnicos de CFT}

El análisis de las mismas refleja la opinión del técnico de CFT y el médico especialista implicados en el estado de preparación para la aplicación del masaje reflexológico podal. De ellos, 1 Medicina General Integral 1 técnico en CF, 1 licenciados en Enfermería para un $33.33 \%$ cada uno

En cuanto a tiempo de experiencia en tratamiento de los pacientes hipertensos, 2 poseen 9 años de experiencias y solo 1 posee 1 año de experiencia

En cuanto a la frecuencia semanal expresan que se realiza ejercicio físico de 2 a 3 veces a la semana en dependencia que no exista otra actividad somos del criterio que en cuba existen diferentes programas de ejercicio físico para el tratamiento del paciente hipertenso, como el que se aplica en las Áreas Terapéuticas realizado por los Doctores Izquierdo Miranda y Morell Rodríguez en 1990, que ha tenido buenos resultados por ser sencillo y de fácil aplicación en la comunidad y seria de fácil ejecución en el programas de la Actividad Física Comunitaria y no se está teniendo en cuenta .

Los principales obstáculos que se presentan en las clases prácticas que imposibilitan que los pacientes se les realicen masaje como adecuada profilaxis y terapéutica después del ejercicio físico son que no contamos con el personal especializado en masaje, además no existen mesas, sillas para la realización del mismo. Que no cuentan con la bibliografía necesaria para consultarla siempre que sea necesario y para la realización de la auto preparación.

La motivación por parte de los adultos a la hora de realizar las actividades físicas no se siente motivada totalmente, la mayoría expresan sentirse cansado. Somos del criterio que falta la base de orientación de la actividad. El principal propósito de una primera etapa que consiste en preparar el paciente para la carga que recibirá en la segunda etapa y proporcionarle los conocimientos básicos sobre su enfermedad la cual debe tener una duración de 2 meses aproximadamente, de acuerdo con la evolución del paciente.

Solo el médico y la enfermera para un $66,66 \%$ conoce los principales motivos o causas de la enfermedad de los pacientes hipertensos que usted atiende pues es el médico que le realiza a la llegada por vez primera a la casa una interrogatorio el cual se guarda en una historia clínica la cual es consultada frecuentemente por la enfermera (poca estabilidad del tratamiento farmacológico, estilo de vida descuidado así como enfermedades asociada como la Diabetes reflejados en los exámenes complementarios 
El 100\% considera que las actividades físicas planificadas para las pacientes hipertensas no son del todo factibles ya que muchos adultos se quejan de que sienten mucho cansancio y prefieren estar realizar otras actividades

El 100\% de los encuestado expresan que si como la Fitoterapia (infusión de caña santa,_Fruto terapia 2 ó 3 vasos de jugo de toronja o naranja agria al día, etc. Esto nos revela la poca cultura que existe acerca de los saberes de las alternativas del masaje terapéutico

Encuesta a adultos

Relación entre grupos de edades y razas de pacientes activos vinculados a programas de la Actividad Física Comunitaria del municipio Güines

Se pudo observar que, de 9 adultos estudiados, $5(55,55 \%)$ se encontraban en las edades comprendidas de 35 a 50 años; asimismo, se encontró que el mayor número de adultos (33,33 $\%)$ correspondía al sexo femenino. En relación con la raza 7 adultos (77,77 \%) pertenecían a la raza negra y sólo $2(22,22 \%)$ es de la raza blanca.

Desde el punto de vista del masaje terapéutico, en el cual resulta necesario accionar sobre aquellos sistemas o partes del cuerpo humano que por su composición y vínculo con el resto del organismo contribuyen a aliviar dolencias, estimular el ritmo de trabajo o reducir tensiones, el microsistema del pie constituye un centro generador de tales efectos hacia los distintos puntos del cuerpo

En el cuerpo humano existen varias zonas o microsistemas que reflejan en pequeñas áreas todo lo que ocurre en el organismo, constituyen reflejos de sistemas y órganos. Son usados con un fin diagnóstico y terapéutico desde tiempos muy antiguos

Se constata que 6 pacientes $(66,3 \%)$ padecían de hipertensión arterial ligera; en 3 pacientes $(33,3 \%)$ se sitúa la hipertensión arterial moderada.

Al inicio del tratamiento con masaje reflexológico podal, los 9 pacientes estudiados mantenían cifras elevadas de tensión arterial sistólica y diastólica, lo que estaba en estrecha relación con algunos factores de riesgo y estilos de vida inadecuados de los mismos.

Para la obtención de la información se utilizaron las historias clínicas de los pacientes, donde se identificó edad, sexo, raza y tipo de hipertensión. La tarjeta de control nos proporcionó determinar el comportamiento de la hipertensión arterial antes de aplicado el tratamiento y después del mismo. El masaje reflexológico podal se les aplicó en 12 sesiones, con una frecuencia de tres veces por semana, en días alternos, con una duración de 15 a 20 minutos en el horario de la mañana. Se midió la tensión arterial al inicio del tratamiento, al final de éste, y de cada sesión, con el mismo esfigmomanómetro y la técnica establecida; las cifras fueron registradas en la tarjeta de control. 
Aquí se demostró cómo existe un mejoramiento de un $100 \%$ en los siguientes síntomas, ojo enrojecido, cara enrojecida y H.T.A y con un $88,88 \%$ en la cefalea, pulso rápido, coincidiendo con otros autores como Mulet Pérez, que plantean la efectividad de la aplicación con la reflexología podal.

Comportamiento de los síntomas después del tratamiento con el masaje reflexológico.

\begin{tabular}{c|c|c|c|c|c|} 
Síntomas & Mejoro & $\%$ & No Mejoro & $\%$ & Total \\
\hline Cefalea & 8 & 88,88 & 1 & 11,11 & \\
\hline Irritabilidad & 7 & 77,78 & 2 & 22,22 & \\
\hline $\begin{array}{c}\text { Cara } \\
\text { enrojecida }\end{array}$ & 9 & 100 & - & - & \multirow{2}{*}{9} \\
\hline $\begin{array}{c}\text { Ojos } \\
\text { enrojecidos }\end{array}$ & 9 & 100 & - & - & \\
\hline $\begin{array}{c}\text { Pulso rápido } \\
\text { y fuerte }\end{array}$ & 8 & 88,88 & 1 & 11,11 & \\
\hline $\begin{array}{c}\text { H.T.A. } \\
\text { fur. }\end{array}$ & 9 & 100 & &
\end{tabular}

Fuente: Elaboración propia.

\section{Conclusiones}

- Síntomas como la Irritabilidad, depresión, cefaleas, Cara enrojecida, Ojos enrojecido Pulso rápido y fuerte encontraron su desaparición con este tipo de tratamiento.

- El masaje reflexológico podal influyó positivamente en el tratamiento de la HTA en el adulto reincorporando a las actividades cotidiana con mayor rapidez, eficiencia y con disminución del riesgo.

\section{Recomendaciones}

- Al INDER se le recomienda la inclusión del masaje reflexológico podal como una alternativa terapéutica más en el tratamiento de la HTA del adulto. 


\section{Referencias bibliográficas.}

Gifford RW. Mitos acerca de la Hipertensión en el anciano. Clin Med North Amer 2005; 71(5):1059-70.

Jafar Th, Atark PC, Pardell H, Guillen F. Hipertensión arterial en el anciano: cambios fisiológicos; conceptos actuales. Madrid: LELHA-SEGG; 2005.

Ministerio de Salud Pública. Enfermedades crónicas no transmisibles. En: Carpeta metodológica. La Habana: Ciencias Médicas; 1998.p. 68-110.

Aldana, S., Vereda, F., Hidalgo-Alvarez, R., \& de Vicente, J. (2016). Facile synthesis of magnetic agarose microfibers by directed selfassembly. Polymer, 93, 61-64.

Bhat, S., Tripathi, A., \& Kumar, A. (2010). Supermacroprous chitosan-agarose-gelatin cryogels. in vitro characterization and in vivo assesment for cartilage tissue engineering. Journal of the Royal Society Interface, 1-15.

Bossis, G., Marins, J., Kuzhir, P., Volkova, O., \& Zubarev, A. (2015). Functionalized microfibers for field-responsive materials and biological applications. Journal of Intelligent Material Systems and Structures, 1-9.

Cortés, J., Puig, J., Morales , J., \& Mendizábal, E. (2011). Hidrogeles nanoestructurados termosensibles sintetizados mediante polimerización en microemulsión inversa. Revista Mexicana de Ingeniería Química., 10(3), 513-520.

Dias, A., Hussain, A., Marcos, A., \& Roque, A. (2011). A biotechnological perspective on the application of iron oxide magnetic colloids modified with polysaccharides. Biotechnology Advances 29, 29, 142-155.

Estrada Guerrero, R., Lemus Torres, D., Mendoza Anaya, D., \& Rodriguez Lugo, V. (2010). Hidrogeles poliméricos potencialmente aplicables en Agricultura. Revista Iberoamericana de Polímeros, 12(2), 76-87.

García-Cerda, L., Rodríguez-Fernández, O., Betancourt-Galindo, R., Saldívar-Guerrero, R., \& Torres-Torres, M. (2003). Síntesis y propiedades de ferrofluidos de magnetita. Superficies y Vacío., 16(1), 28-31.

Ilg, P. (2013). Stimuli-responsive hydrogels cross-linked by magnetic nanoparticles. Soft Matter, 9, 3465-3468.

Lewitus, D., Branch, J., Smith, K., Callegari, G., Kohn, J., \& Neimark, A. (2011). Biohybrid carbon nanotube/agarose fibers for neural tissue engineering. Advanced Functional Materials, 21, 2624-2632. 
Lin, Y.-S., Huang, K.-S., Yang, C.-H., Wang, C.-Y., Yang, Y.-S., Hsu, H.-C., . . Tsai, C.W. (2012). Microfluidic synthesis of microfibers for magnetic-responsive controlled drug release and cell culture. PLoS ONE, 7(3), 1-8.

Ruiz Estrada, G. (2004). Desarrollo de un Sistema de liberación de fármacos basado en nanopartículas magnéticas recubiertas con Polietilénglicol para el tratamiento de diferentes enfermedades. Madrid: Universidad Autónoma de Madrid. Departamento de Física Aplicada.

Song, J., King, S., Yoon, S., Cho, D., \& Jeong, Y. (2014). Enhanced spinnability of narbon nanotube fibers by surfactant addition. Fiberes and Polymers, 15(4), 762-766.

Tartaj, P., Morales, M., González-Carreño, T., Veintemillas-Verdaguer, S., \& Serna, C. (2005). Advances in magnetic nanoparticles for biotechnology applications. Journal of Magnetism and Magnetic Materials, 290, 28-34.

Wulff-Pérez , M., Martín-Rodriguez, A., Gálvez-Ruiz, M., \& de Vicente, J. ( 2013 ). The effect of polymer surfactant on the rheological properties of nanoemulsions. Colloid and Polymer Science, 291, 709-716.

Zamora Mora, V., Soares, P., Echeverria, C., Hernández , R., \& Mijangos, C. (2015). Composite chitosan/Agarose ferrogels for potential applications in magnetic hyperethermia. Gels., 1, 69-80. 


\section{PARA CITAR EL ARTÍCULO INDEXADO.}

González Pérez, E. R., \& González Paneque, A. (2018). El masaje reflexológico podal como medio alternativo en el tratamiento de adultos hipertensos. Anatomía Digital, 1(1), 6-15. https://doi.org/10.33262/anatomiadigital.v1i1.1037

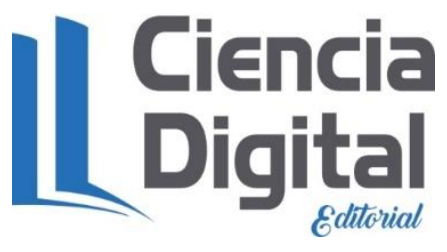

El artículo que se publica es de exclusiva responsabilidad de los autores y no necesariamente reflejan el pensamiento de la Revista Anatomía Digital.

El artículo queda en propiedad de la revista y, por tanto, su publicación parcial y/o total en otro medio tiene que ser autorizado por el director de la Revista Anatomía Digital.
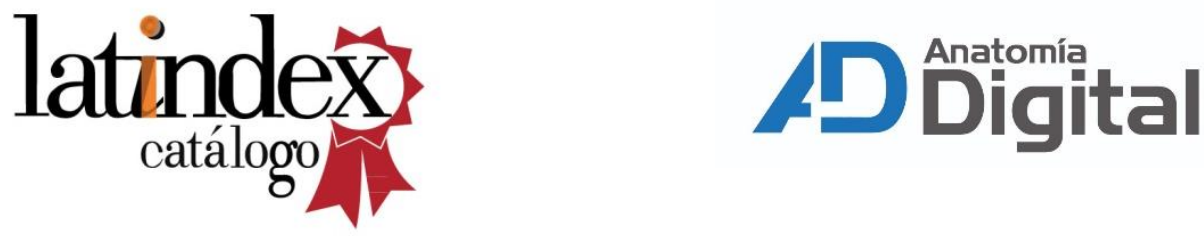